\title{
Towards Revised Step IV MICE Optics in the Absence of M1 SSD
}

R. Bayes (Glasgow U.) , J.S. Berg (Brookhaven), V. Blackmore, C. Hunt (Imperial Coll., London), A. Liu(Fermilab) , J. Pasternak (Imperial Coll., London), C.T. Rogers (Rutherford)

\section{Introduction}

During magnet commissioning in September 2015, the leads on coil M1 of the downstream spectrometer solenoid failed. The coil will not be operational for MICE Step IV. Revised optics settings for the Step IV data taking are reviewed.

\subsection{Aims of MICE Step IV}

MICE Step IV aims to

- Measure material properties of $\mathrm{lH}_{2}$ and $\mathrm{LiH}$.

- Observe normalised transverse emittance reduction.

The MICE collaboration seeks to make measurements, over a range of momenta, a variety of different optical $\beta$ functions and emittances, with and without field flips available in the magnets.

\subsection{The MICE Lattice}
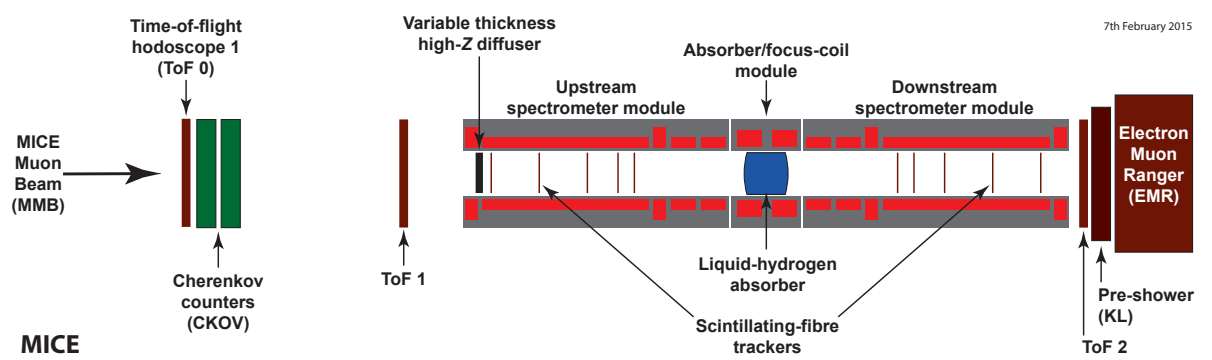

Fig. 1: Schematic of MICE at Step IV.

The MICE lattice was designed to provide a good performance over these various settings. A schematic of the MICE channel is shown in figure 1. The function of the main lattice elements are described below. 
- The diffuser provides a variable thickness scatterer in the beam, enabling experimentalists to select different emittances.

- The outer 3 coils of the spectrometer modules are, from outermost to innermost, End2 coil, Center coil and End1 coil (ECE). The Center coil provides a solenoidal field to enable helix fitting in the trackers. End2 and End1 are trim coils used to make the field more constant in the tracker region.

- The inner 2 coils of the spectrometer modules are Match2 and Match1. The Match coils provide matching from the constant solenoidal field to the absorber region. Two coils enable independent selection of $\beta$ and $d \beta / d z$.

- The Focus coil provides a final focus on the absorber.

The coil geometries used in this note are summarised in [1].

\subsection{Measurements in the MICE Lattice}

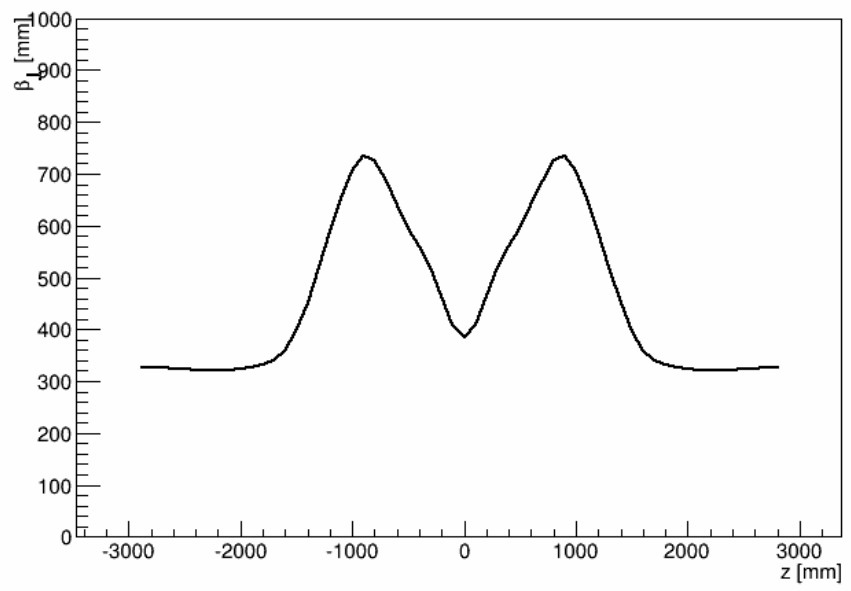

Fig. 2: Baseline $\beta$ function as a function of $\mathrm{z}$, with M1 and M2 available at upstream and downstream spectrometers.

Measurement of emittance reduction requires that a beam with emittance greater than the equilibirum emittance is transported from the upstream Tracker Reference Plane (TRP), where the beam is measured, through the absorber to the downstream TRP. This requires a small $\beta$ function and large acceptance, with minimal non-linear emittance growth. An additional, indirect, method of measuring emittance reduction can be made by projecting particles measured at the TRP to either side of the absorber, calculating emittance before and after. 
Measurements of material physics processes are insensitive to beam optics and limited by statistics. Therefore, maximising the number of particles transported to the absorber and downstream TRP improves these measurements.

A baseline optical $\beta$ function and corresponding $B_{z}$ is shown in figure 2 . The following constraints were applied in order to find the optics solution.

- The $\beta$ function is symmetric about the absorber.

- The $\beta$ function is as flat as possible in the Match coil region.

- The $\beta$ function is flat in the solenoid region.

While these properties are not essential, they result in a good acceptance and suppress high order non-linearities that are present for such a high emittance beam. These non-linearities can result in emittance growth that is significant with respect to the emittance reduction produced at the absorber.

\subsection{Baseline-Like Optics Settings Without M1}

In the absence of M1 the range of available optics solutions is greatly reduced if the requirement that $\beta$ is constant in the spectrometer and $\beta$ is symmetric about the absorber is maintained.

In figure 3 a selection of available $\beta$ functions at the absorber are shown. The fractional change in RMS emittance is shown as a function of $\beta$ in figure 4 . When the emittance change is less than 0 , the beam is cooled. For acceptances typical of the MICE lattice, $\beta$ functions below $1 \mathrm{~m}$ are required. The $\beta$ function in all cases is too high to transport a beam with the MICE equilibrium emittance through the cooling channel, so in these circumstances MICE would heat the beam rather than cool it.

It is clear that in order to make a lattice that cools, the constraints must be relaxed.

\section{Material Physics Measurements}

It is noted that measurement of material physics processes in the MICE absorbers and indirect measurement of emittance reduction is still possible, even without further optimisation.

For example, during data taking on Wednesday 7th October 2015, SSU End1 coil, Center coil and End2 coil (ECE) were powered. SSD was not powered. In this configuration, 13000 particles per hour traversed the experiment to TOF2 running in a $200 \mathrm{MeV} / \mathrm{c}$ muon mode. This corresponds to more than $20 \%$ of the hits in TOF1. It is expected that the transport efficiency will be improved if SSD ECE were powered, with further improvements achieved by powering the match coils and focus coils and reoptimising the beamline for the available coils.

This should be compared with 2.3e6 and 1.1e6 events that MuScat measured on $109 \mathrm{~mm}$ and $159 \mathrm{~mm}$ liquid Hydrogen targets respectvely [2]. To achieve comparable statistics to MuScat, assuming no further rate optimisation, would 

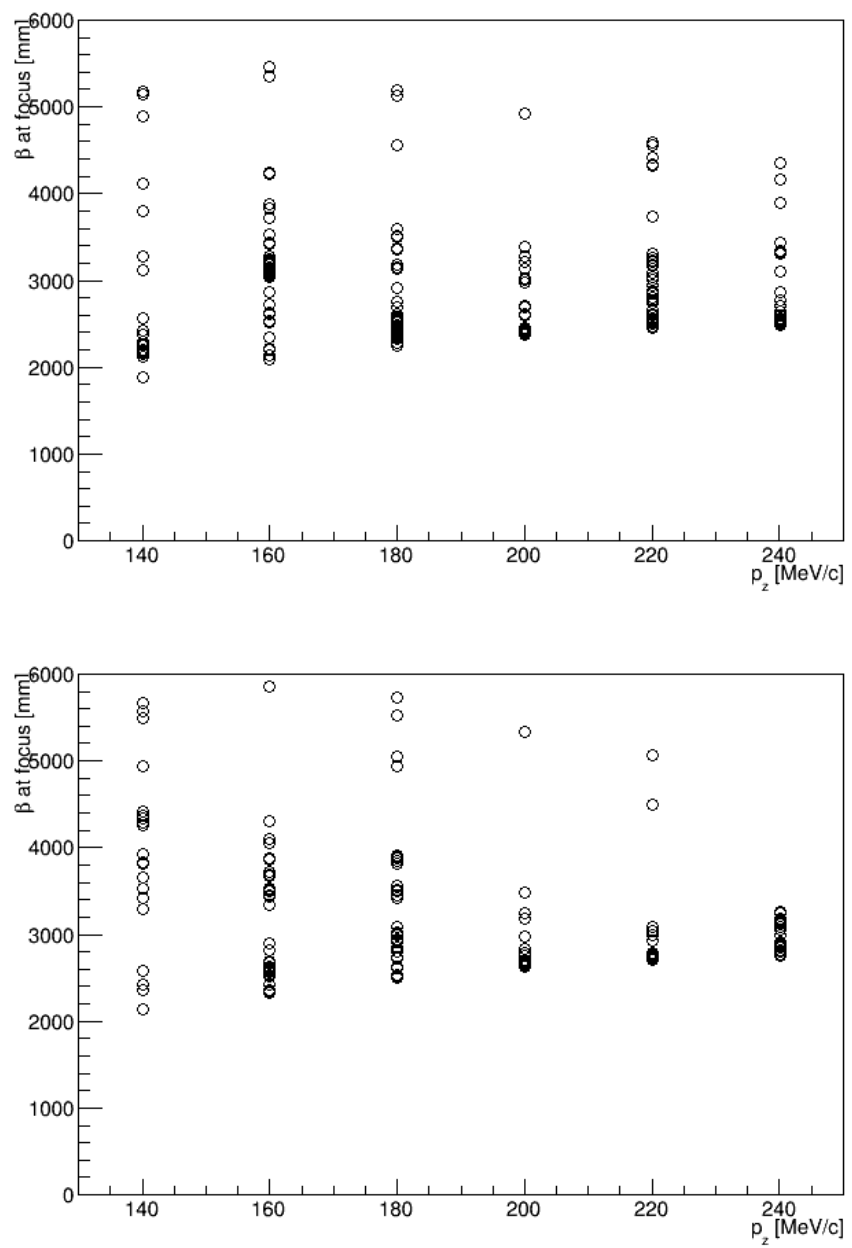

Fig. 3: Available $\beta$ at the focus coil, given "baseline" constraints on MICE (top) in flip mode, with field antisymmetric about the lattice centre, and (bottom) in solenoid mode, with field symmetric about the lattice centre. 


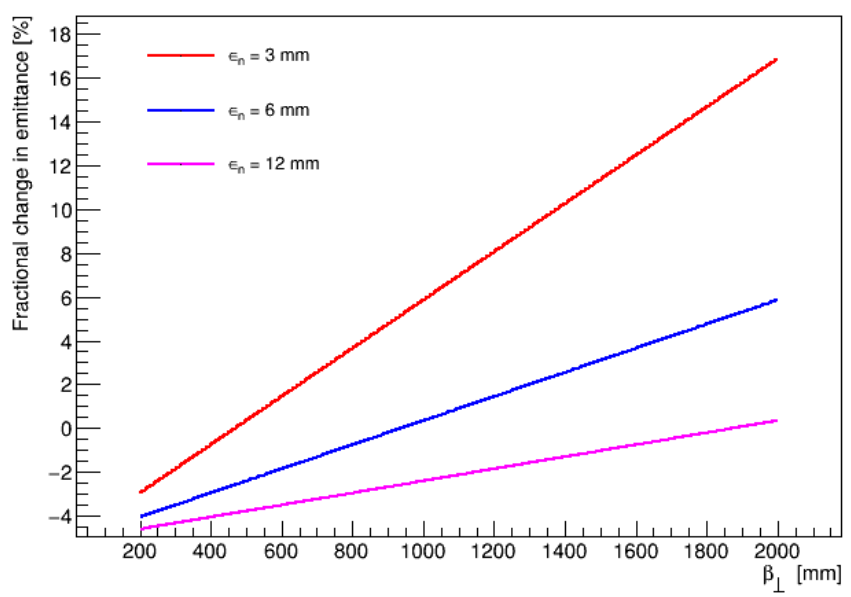

Fig. 4: Fractional emittance reduction for various input emittances and optical $\beta$ functions. $200 \mathrm{MeV} / \mathrm{c}$ muons are considered incident on a $65 \mathrm{~mm}$ Lithium Hydride absorber. Small angle approximations and thin absorber approximations are assumed.

require 1-2 weeks of data taking on each absorber. Note that the MICE beam has a larger momentum spread than the MuScat beam and this would need to be managed in the analysis.

MICE will also make a measurement of multiple scattering with no fields in the trackers. In this configuration, resolutions in transverse angle are expected to be better, but rate and resolutions in energy (required for particle selection) are expected to be worse. Clearly such a configuration is unaffected by the presence of fields.

\section{Available Optics}

In order to achieve an optics that can reduce emittance in MICE, one of the boundary conditions described in Section 1.3 must be relaxed. Various different constraints are relaxed and the resultant optics solutions are discussed.

\subsection{Asymmetric Optics with $\beta$ Constant in Tracker}

In this section the requirement that the optics is symmetric about the absorber is relaxed. The constraint that $\beta$ is constant in the spectrometer is maintained. In order to achieve sufficient focussing for emittance reduction at 200 (and 240) $\mathrm{MeV} / \mathrm{c}$, the magnetic field in the solenoids must be reduced. Linear optics solutions are available that may exhibit cooling at $140 \mathrm{MeV} / \mathrm{c}$ without reducing 
the magnetic field in the solenoids.

In figure 5 the optical $\beta$ function at the absorber is shown as a function of magnetic field in the spectrometer solenoids. Here the upstream and downstream spectrometer solenoid fields are constrained to be identical and $\beta$ in the spectrometers is held constant.

In order to recover a cooling optics at $200 \mathrm{MeV} / \mathrm{c}$, the field in the spectrometer solenoid must be degraded to around $1.2 \mathrm{~T}$. There are two consequences to this; the performance of the tracker is reduced; and the acceptance in the tracker region is reduced. The tracker resolution is discussed in Section 4.

The acceptance in a $1.1 \mathrm{~m}$ long constant field region is shown in figure 6 as a function of momentum and magnetic field. Here the $150 \mathrm{~mm}$ fiducial radius of the tracker has been assumed. Note that in the lattices considered here, the tracker is the limiting aperture; previously the region around the match coil was limiting.

\subsection{Symmetric Lattice with Beta-Beating in Spectrometer}

Instead of relaxing the symmetry of the optics in the cooling channel, one may relax the requirement that $\beta$ is constant in the spectrometer region. The field in the spectrometer is maintained at $4 \mathrm{~T}$ and the optics are required to be symmetric about the absorber, with the field either symmetric or antisymmetric.

In figure 7 the minimum achievable $\beta$ with non-constant $\beta$ in the spectrometer region is shown as a function of $\beta$ and $\alpha$ at the tracker reference plane.

One consequence of a non-constant $\beta$ in the spectrometer is that the acceptance in the tracker region will be reduced. The acceptance in a $1.1 \mathrm{~m}$ long constant field region is shown in figure 8 as a function of the initial $\beta$ and $\alpha$. The time-reversal symmetry ensures that the acceptance will be the same upstream and downstream, barring a sign flip in alpha.

\subsection{Asymmetric Lattice with Beta-Beating in Spectrometer}

It is also possible to abandon both the constraint of a symmetric optics and the boundary condition that $\beta$ is constant in the solenoid.

In this general case, the lattice is optimised by minimising the ratio of equilibrium emittance to acceptance. In the paraxial, thin absorber approximation the acceptance is given by

$$
A_{\max }=\frac{r^{2} p_{z}}{\beta_{\perp} m}
$$

and the equilibrium emittance is given by

$$
\epsilon_{\text {eqm }}=\frac{1}{2 m} \frac{13.6^{2}}{L_{R}} \frac{\beta_{\perp}}{\beta_{r e l}<d E / d z>} .
$$

The ratio is minimal when the ratio $\beta_{\perp}(z) \beta_{\perp}$ (absorber) $/ r^{2}(z)$ is minimal for all $z$. A minimising routine was used to find optimum conditions for the lattice given these constraints. Initial conditions were chosen randomly and 


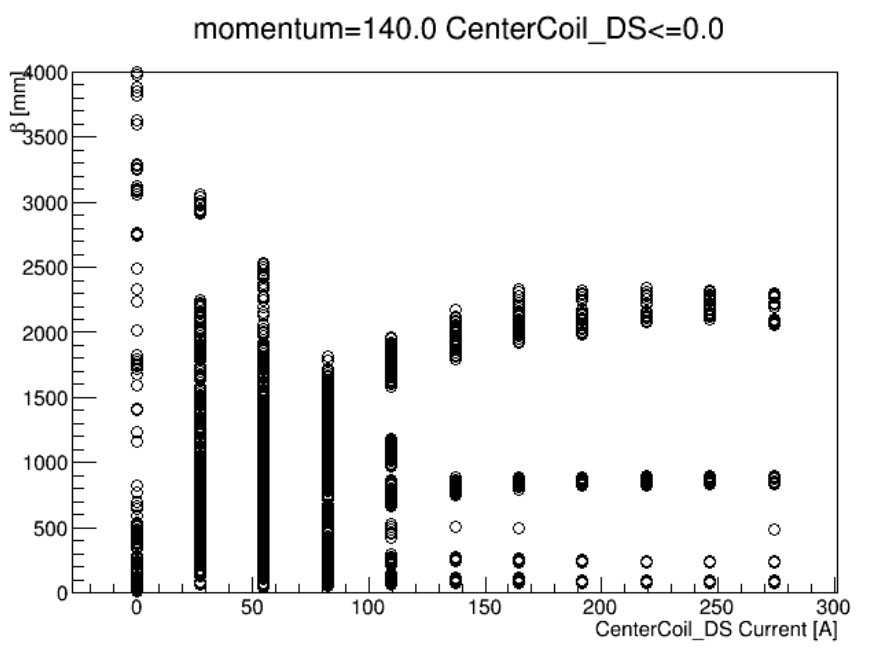

momentum $=200.0$ CenterCoil_DS $<=0.0$

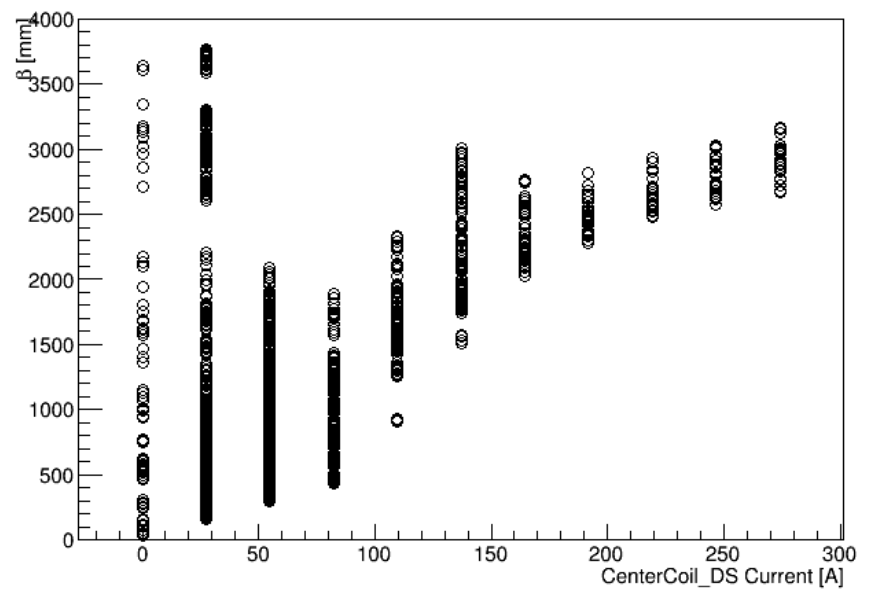

Fig. 5: Flip mode optical functions at the absorber, as a function of CenterCoil current for (top) $140 \mathrm{MeV} / \mathrm{c}$ (bottom) $200 \mathrm{MeV} / \mathrm{c}$. At $4 \mathrm{~T}$ the downstream Center Coil, CenterCoil_DS, operates at 274 A.

R. Bayes (Glasgow U.), J.S. Berg (Brookhaven), V. Blackmore, C. Hunt (Imperial Coll., London), A. Liu(Fermilab), J. Pasternak (Imperial Coll., London), C.T. Rogers (Rutherford) 


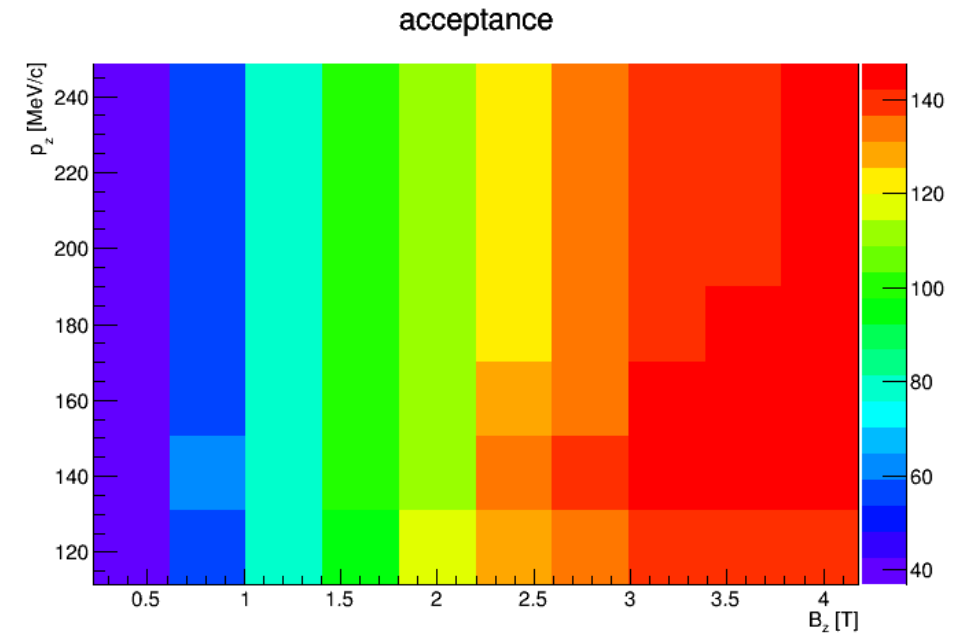

Fig. 6: Acceptance $[\mathrm{mm}]$ in a tracker as a function of $B_{z}$ and $p_{z}$. The $150 \mathrm{~mm}$ tracker fiducial volume and constant optical $\beta$ function is assumed.

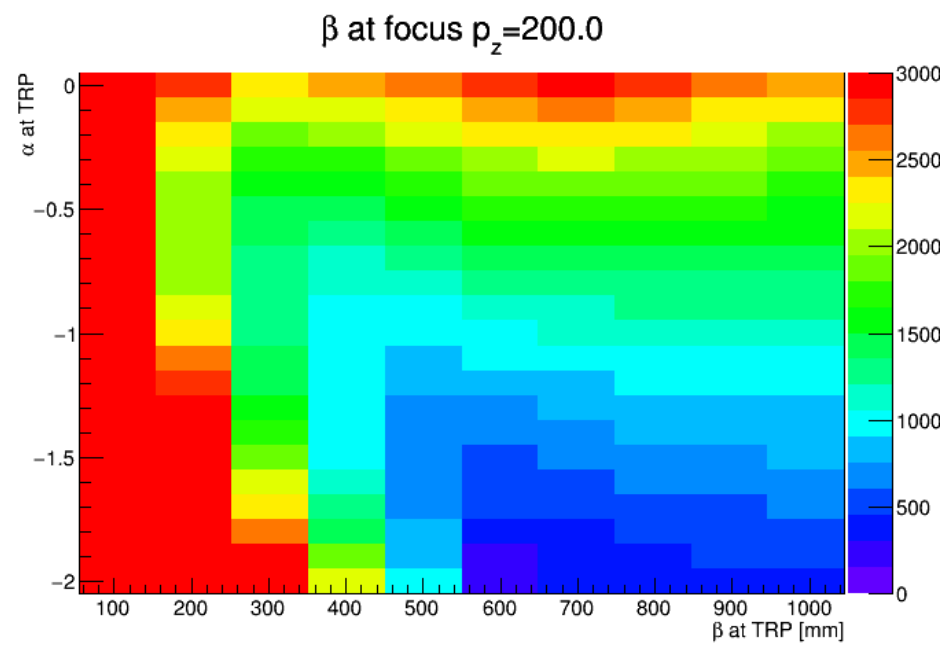

Fig. 7: Minimum achievable $\beta$ with non-constant $\beta$ in the spectrometer region as a function of $\beta$ and $\alpha$ at the tracker reference plane. Magnets were in an antisymmetric configuration and beam was at $200 \mathrm{MeV} / \mathrm{c}$. 


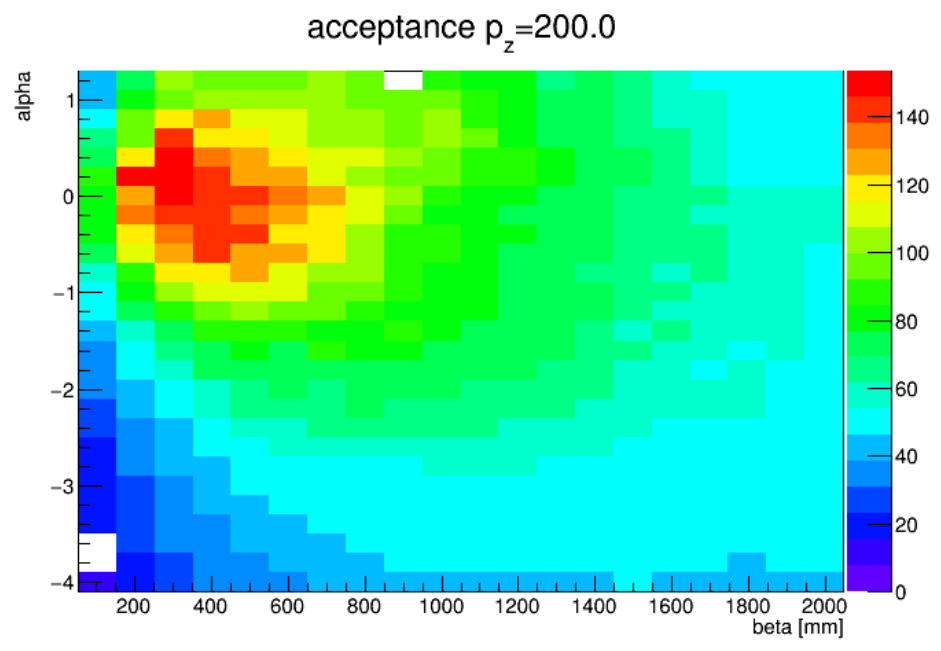

Fig. 8: Acceptance in a tracker as a function of $\beta$ and $\alpha$ at the tracker reference plane. Acceptance was found by tracking a beam through a constant $B_{z}$ region and removing particles that had radius more than $150 \mathrm{~mm}$, the fiducial volume of the tracker. Particles were binned in acceptance and the bin where the number of surviving particles in an acceptance bin was less than $50 \%$ was considered the acceptance. All particles in the beam had $p_{\text {tot }}$ of $200 \mathrm{MeV} / \mathrm{c}$.

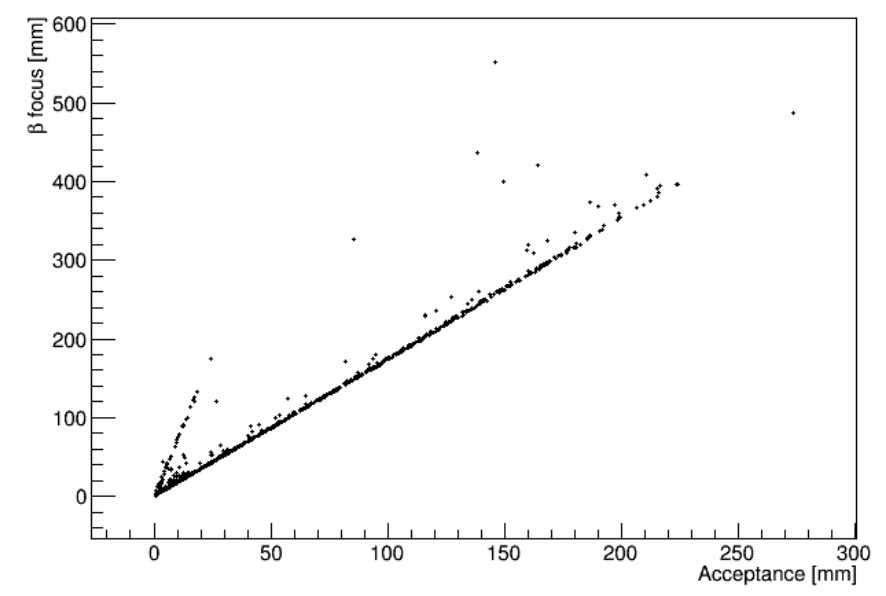

Fig. 9: Minimum achievable $\beta$ at the absorber focus as a function of $\beta$ and $\alpha$ at the tracker reference plane. Magnets were in an antisymmetric configuration and beam was at $200 \mathrm{MeV} / \mathrm{c}$. 
the optimising routine was allowed to converge. The population of minimal solutions is shown in figure 9 as a function of $\beta$ at the absorber and minimum scraping aperture acceptance.

It is noted that optimal solutions have the Focus coil running at the maximum achievable current, M2 in SSD operating at the maximum achievable current and the limiting aperture is at the downstream end of the downstream tracker.

\section{Detector Resolutions with Reduced Spectrometer Field}
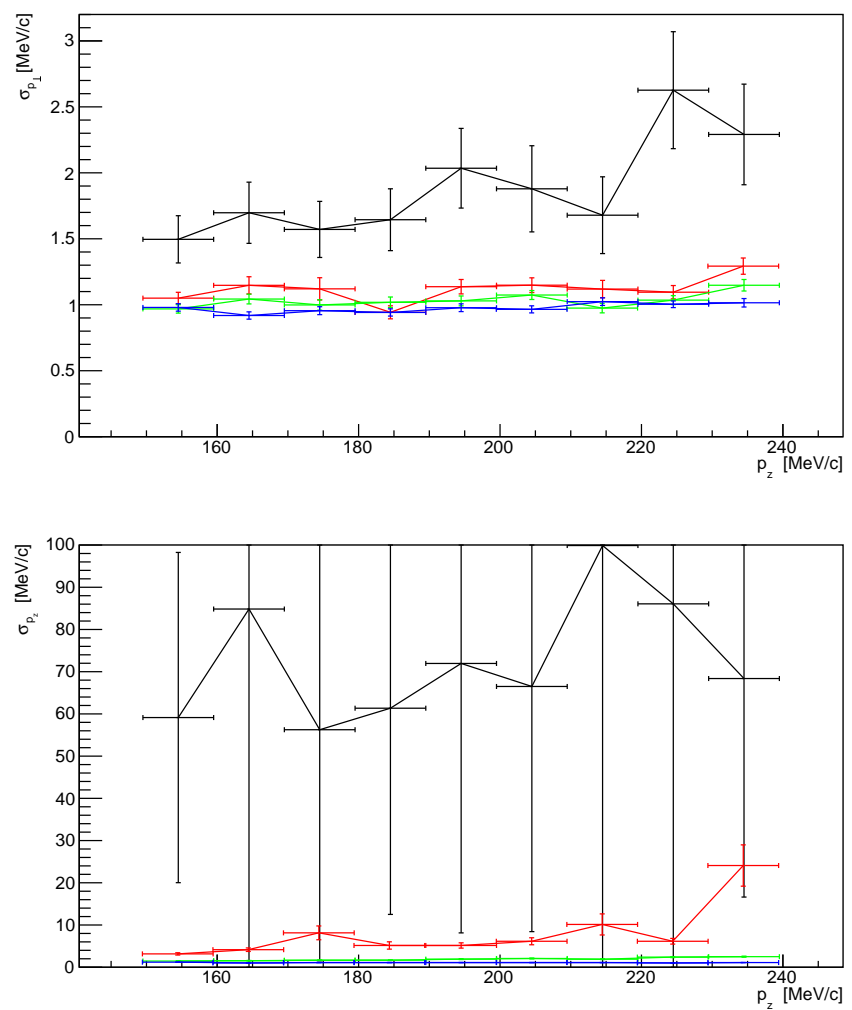

Fig. 10: Tracker resolution in $p_{z}$ and $p_{t}$ for different tracker magnetic fields: (black) $1 \mathrm{~T}$; (red) $2 \mathrm{~T}$; (green) $3 \mathrm{~T}$; and (blue) $4 \mathrm{~T}$.

The tracker performance is likely to be compromised by reduced fields. The tracker reconstruction routine relies on a helix fit to reconstruct particle momentum. In a reduced field the helix radius increases but the wavelength of the helix also increases. Tracks make only a fraction of a turn in the tracker length 


\begin{tabular}{l|cccc|}
$\begin{array}{l}\text { Lattice } \\
\text { Index }\end{array}$ & $\begin{array}{c}\mathrm{p} \\
\mathrm{MeV} / \mathrm{c}\end{array}$ & $\begin{array}{c}\beta_{a b s} \\
\mathrm{~mm}\end{array}$ & $\begin{array}{c}\beta_{\text {in }} \\
\mathrm{mm}\end{array}$ & $\alpha_{\text {in }}$ \\
\hline 1 & 200 & 447 & 1102 & 0 \\
\hline 2 & 200 & 574 & 600 & -1.5 \\
3 & 200 & 687 & 600 & -1.5 \\
\hline 4 & 200 & 411. & 743.19 & -0.748
\end{tabular}

Tab. 1: Summary of beam parameters for lattices simulated below.

and this can make reconstruction challenging.

In order to measure transverse emittance, measurement of $x, p_{x}, y$ and $p_{y}$ is required. Measurement of $p_{z}$ is required in order to select particles with the appropriate momentum for the lattice, as the MICE beamline provides muons with a too-large momentum spread. Measurement of $p_{z}$ may also improve PID performance by improving on the momentum selection that already occurs in dipole D2. The momentum acceptance is discussed in section 5 but it is a few $\mathrm{MeV} / \mathrm{c}$.

The simulated tracker resolution in transverse momentum $p_{t}$ and longitudinal momentum $p_{z}$ is shown in figure 10. The $p_{t}$ resolution is acceptable in these circumstances but the $p_{z}$ resolution is quite poor. The resolution in $x$ and $y$ is not affected by the magnetic field.

Selection of an appropriate momentum spread can be made by using Timeof-flight and range of energy deposited in the EMR. The time-of-flight has excellent momentum resolution for the particles under consideration. Energy straggling in the diffuser is expected to contribute to uncertainty in the momentum measurement which may limit the usability of the diffuser in low spectrometer field configuration. Energy straggling in the absorber and other materials will contribute around $4 \mathrm{MeV} / \mathrm{c}$ momentum spread in the beam. Time of flight dependence on amplitude is also significant for high emittance beams, but this can be estimated using a global fitting algorithm.

The EMR can also provide momentum reconstruction by measuring particle range in the detector. This gives momentum resolutions of around $10 \mathrm{MeV} / \mathrm{c}$ $[3]$.

\section{Tracking Performance Comparison and Normalised Emittance Reduction}

Several optics solutions are studied and are summarised in Table 1 and 2. The four lattices have the following optimisation properties:

1. $\beta$ is constant in the solenoid field; the lattice optics are allowed to be asymmetric and the tracker region of the spectrometer is at $1.2 \mathrm{~T}$.

2. $\beta$ beats in the solenoid field; the lattice optics are constrained to be symmetric, and the tracker field is maintained at $4 \mathrm{~T}$. A lower $\beta$ function 


\begin{tabular}{l|cccc|ccc}
$\begin{array}{l}\text { Lattice } \\
\text { Index }\end{array}$ & $\begin{array}{c}\mathrm{E} 2 \\
{[\mathrm{~A}]}\end{array}$ & $\mathrm{CC}$ & $\mathrm{E} 1$ & $\mathrm{M} 2 \mathrm{US}$ & $\mathrm{M} 1 \mathrm{US}$ & $\mathrm{FC}$ & $\mathrm{M} 2 \mathrm{DS}$ \\
\hline 1 & 75.90 & 82.20 & 70.20 & 107.8 & 250.9 & 225.0 & -231.6 \\
\hline 2 & 253.00 & 274.00 & 234.00 & 279.99 & 0.0 & 189.45 & -279.99 \\
3 & 253.00 & 274.00 & 234.00 & 255.05 & 0.0 & 149.26 & -255.05 \\
\hline 4 & 253.00 & 274.00 & 234.00 & 0.0 & 277.53 & 224.83 & -279.71
\end{tabular}

Tab. 2: Summary of coil currents for lattices simulated below. In all cases the ECE downstream coils were simulated with the same current as the ECE upstream currents bar a factor -1 . The downstream Focus coil was simulated with the same current as the upstream focus coil bar a factor -1. The downstream Match coil 1 was not powered. Coil geometries are taken from [1].

option is selected.

3. $\beta$ beats in the solenoid field; the lattice optics are constrained to be symmetric, and the tracker field is maintained at $4 \mathrm{~T}$. A higher $\beta$ function option is selected.

4. $\beta$ beats in the solenoid field; the lattice optics are asymmetric, and the tracker field is maintained at $4 \mathrm{~T}$.

Beams were simulated travelling from the upstream tracker reference plane to the downstream tracker reference plane. The absorber was included for some simulations but the tracker helium window and associated helium was not included. This may introduce some additional heating. Particles striking the apertures were killed. Emittances are computed only for particles that make it through to the downstream spectrometer.

In figure 11 the optical $\beta$ is calculated for a selection of paraxial, monochromatic beams propagating through each lattice, with material physics switched off. For asymmetric lattices the focus is shifted upstream of the absorber as more focussing is available in this region.

Beams with a $5 \mathrm{MeV} / \mathrm{c}$ momentum spread and $6 \mathrm{~mm}$ transverse emittance are tracked and the resultant emittance evolution is shown in 12 . The effect of aberrations is clearly visible. Optical emittance growth is observed to be significant with respect to the cooling effect of the absorber. In the case of lattice 2, optical emittance growth is more significant than the cooling effect induced by the absorber i.e. the beam is heated.

The cooling performance as a function of input beam emittance and momentum spread is shown in figure 13. Lattice 2 performance is poor due to optical aberrations and it barely cools. Lattices 3 and 4 give at best $2 \%$ cooling. Around half of the cooling effect is disguised by optical emittance growth. Lattice 2 does not cool at all. Lattice 4 shows the least sensitivity to chromatic aberrations but a stronger response to spherical aberrations. 
$\mathrm{n}: 10000$ physics: none $\sigma(\mathrm{p}): 1 \mathrm{e}-06 \in: 0.001$

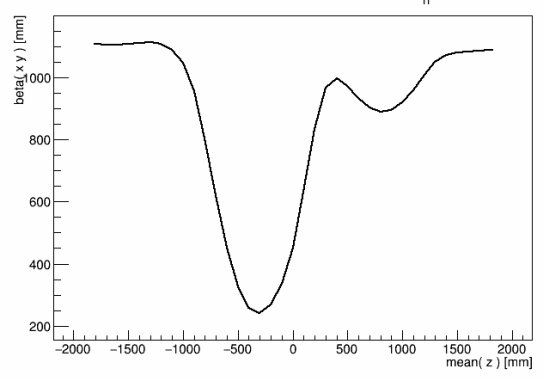

n: 10000 physics: none $\sigma(p): 1 e-06 \epsilon_{n}: 0.001$

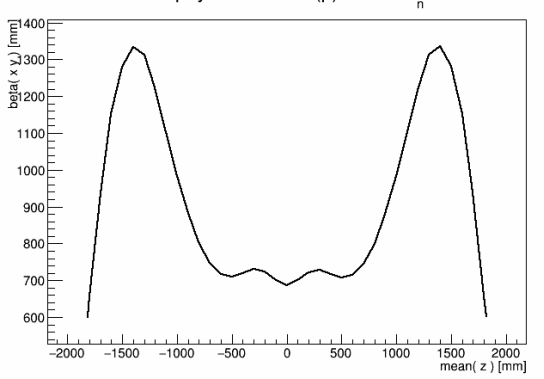

n: 10000 physics: none $\sigma(p): 1 \mathrm{e}-06 \in_{n}: 0.001$

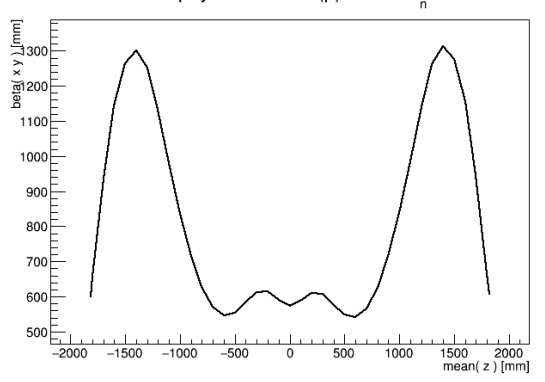

n: 10000 physics: none $\sigma(p): 1 e-06 \in \epsilon_{n}: 0.001$

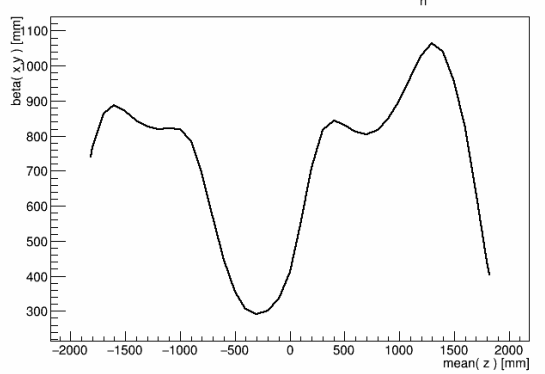

Fig. 11: Paraxial $\beta$ function for the lattices. (Top left) lattice 1 (top right) lattice 2 (bottom left) lattice 3 (bottom right) lattice 4 .

\section{Conclusions}

A revised optics has been studied following failure of the Match1 coil in the downstream spectrometer solenoid. Several situations have been studied.

- $\beta$ is constant in the solenoid field; the lattice optics are allowed to be asymmetric and the tracker region of the spectrometer is derated.

- $\beta$ beats in the solenoid field; the lattice optics are constrained to be symmetric, and the tracker field is maintained at $4 \mathrm{~T}$.

- $\beta$ beats in the solenoid field; the lattice optics are asymmetric, and the tracker field is maintained at $4 \mathrm{~T}$.

Of the four lattices that have been simulated in detail, three show cooling. Further lattice optimisation work is ongoing to reduce the effects of nonlinearities in the optics.

Non-linear matching and beam selection routines have been developed as part of the studies on the wedge absorber and these have be applied to reduce non-linear emittance growth. They can be applied to the current situation also.

MICE will measure energy loss and multiple Coulomb scattering. Without any optimisation a large throughput has been achieved in the cooling channel that can provide a sufficient statistic to be competitive with MuScat, albeit with a larger momentum spread, which MICE will measure. 

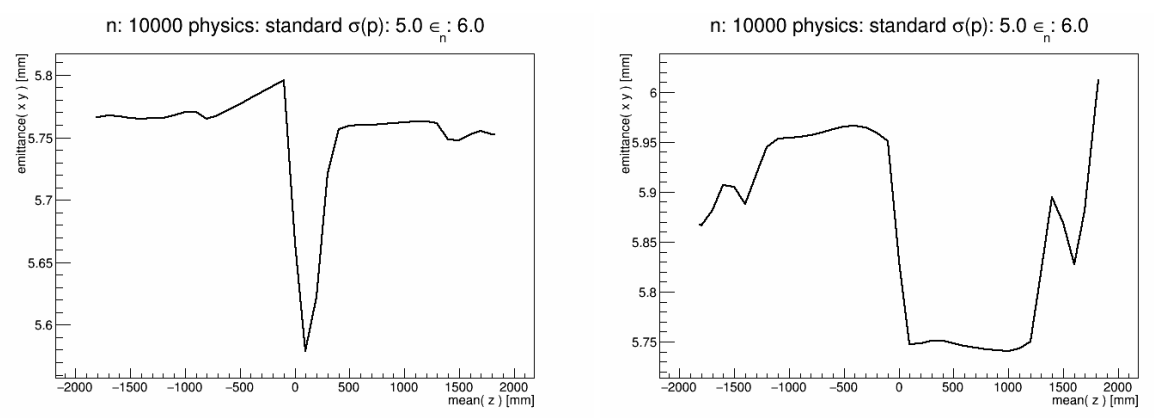

n: 10000 physics: standard $\sigma(p): 5.0 \epsilon_{n}: 6.0$

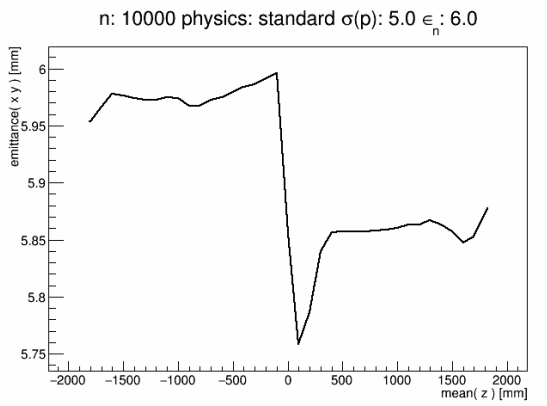

Fig. 12: Emittance change as a function of z. (Top left) lattice 1 (top right) lattice 2 (bottom left) lattice 3 (bottom right) lattice 4 .

\section{References}

[1] As-Built Solenoid Coil Dimensions and Locations in MICE Step IV, V.Blackmore, J. Tarrant, MICE Note 464, 2015.

[2] The scattering of muons in low Z materials, D. Attwood et al, NIM B $251 \mathrm{p}$ 41-55 arXiv:hep-ex/0512005v1, 2005.

[3] Electron-Muon Ranger: Performance in the MICE beam, F. Drielsma et al., MICE Note 466, 2015. 

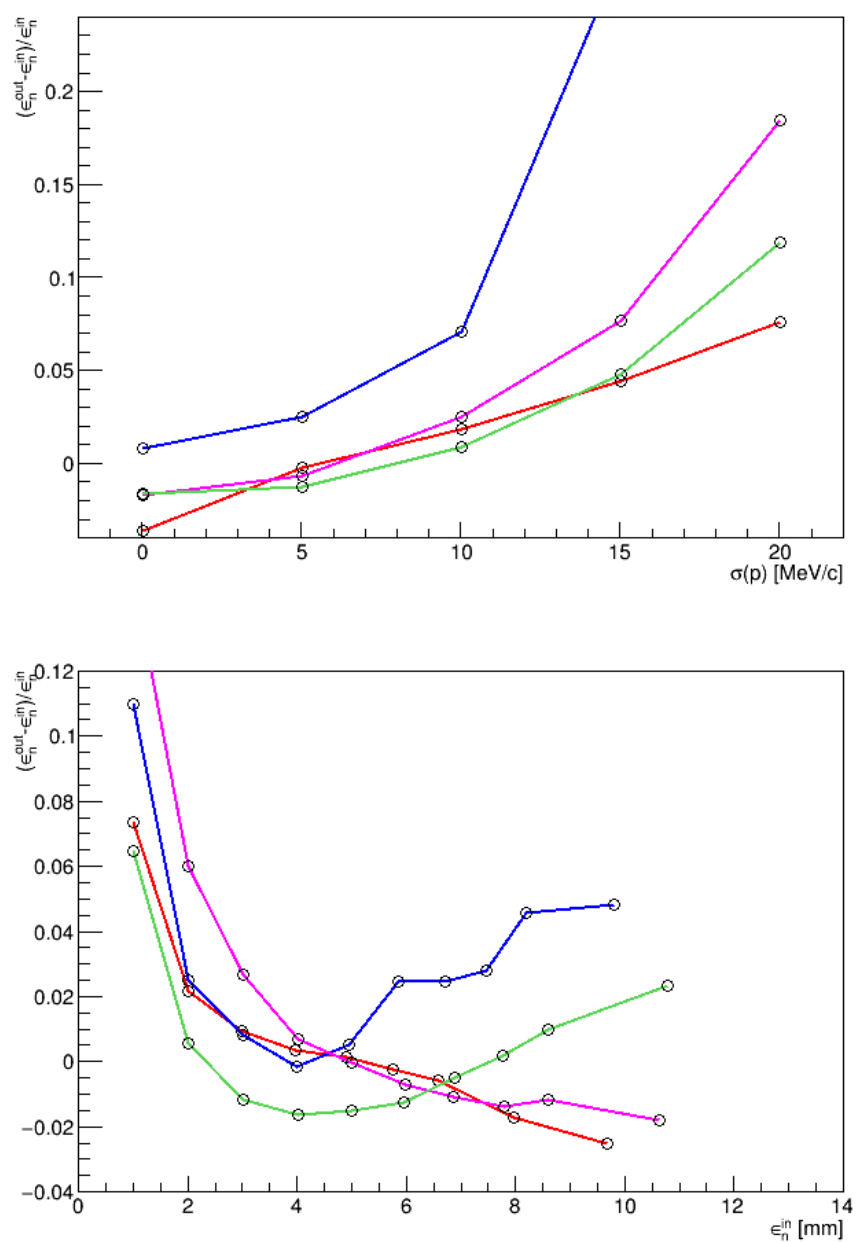

Fig. 13: (Top) Emittance change as a function of momentum spread for an input beam with $6 \mathrm{~mm}$ emittance. (Bottom) emittance change as a function of emittance for an input beam with $5 \mathrm{MeV} / \mathrm{c} \sigma(p)$. Lattice 1 is shown in red, lattice 2 in blue, lattice 3 in pink and lattice 4 in green. 